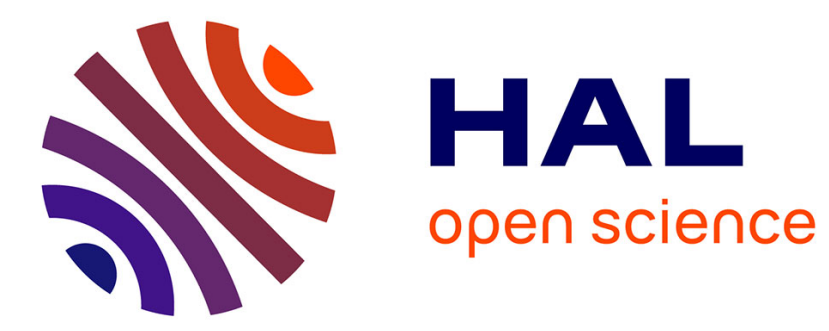

\title{
Sensitivity Analysis Applied to Decision Making in Multiobjective Evolutionary Optimization
}

Sergio Luciano Avila, A.C. Lisboa, Laurent Krähenbühl, Walter P. Carpes, Joao Antonio Vasconcelos, R. R. Saldanha, Ricardo H. C. Takahashi

\section{- To cite this version:}

Sergio Luciano Avila, A.C. Lisboa, Laurent Krähenbühl, Walter P. Carpes, Joao Antonio Vasconcelos, et al.. Sensitivity Analysis Applied to Decision Making in Multiobjective Evolutionary Optimization. IEEE Transactions on Magnetics, 2006, 42 (4), pp.1103-1106. 10.1109/TMAG.2006.871447 . hal00111247

\section{HAL Id: hal-00111247 \\ https://hal.science/hal-00111247}

Submitted on 4 Nov 2006

HAL is a multi-disciplinary open access archive for the deposit and dissemination of scientific research documents, whether they are published or not. The documents may come from teaching and research institutions in France or abroad, or from public or private research centers.
L'archive ouverte pluridisciplinaire HAL, est destinée au dépôt et à la diffusion de documents scientifiques de niveau recherche, publiés ou non, émanant des établissements d'enseignement et de recherche français ou étrangers, des laboratoires publics ou privés. 


\title{
Sensitivity Analysis Applied to Decision Making in Multiobjective Evolutionary Optimization
}

\author{
S. L. Avila ${ }^{1,2}$, A. C. Lisboa $^{3}$, L. Krähenbühl ${ }^{2}$, W. P. Carpes, Jr. ${ }^{1}$, J. A. Vasconcelos ${ }^{3}$, R. R. Saldanha ${ }^{3}$, and \\ R. H. C. Takahashi ${ }^{3}$ \\ ${ }^{1}$ Universidade Federal de Santa Catarina, Florianopolis 88040-970 Brazil \\ ${ }^{2}$ Ecole Centrale de Lyon, Ecully 69130 France \\ ${ }^{3}$ Universidade Federal de Minas Gerais, Belo Horizonte 31160-030 Brazil
}

\begin{abstract}
The result of a multiobjective evolutionary optimization is an efficient solution set surrounded by other candidate solution points. To choose a final solution, we can perform a sensitivity study. Applying this methodology, disturbances that occur in real-world design problems are not neglected. This paper presents an easy way to perform the sensitivity analysis directly from the data generated from a multiobjective stochastic optimization process. No additional function evaluation is required. As an example, we have solved some optimization problems concerning electromagnetic devices.
\end{abstract}

Index Terms-Multiobjective evolutionary optimization, sensitivity analysis.

\section{INTRODUCTION}

$\mathbf{I}$ N ENGINEERING design, sensitivity analysis verifies how stable is the performance of a designed device, since deviations from project specifications are unavoidable in real world. The sources of disturbances may be inaccurate construction or changes on operational conditions (due to mechanical deformation forces, for example).

Electromagnetic optimization problems are often computationally expensive, nonlinear and composed by conflicting goals. Multiobjective Genetic Algorithms (MGAs) are robust, stochastic-based methods which can handle the common features of electromagnetic problems [1]-[3]. Like any multi-objective algorithm, the MGA must provide a meaningful set of samples from the group of efficient solutions, called Pareto-optimal front, which represents the tradeoff among objective functions. The decision maker then chooses one final solution from this set. In this work, the decision maker is supported by sensitivity analysis information, in order to quantify the solution stability.

Among several possible sensitivity metrics, the Lipschitz constant has been used. It is defined as the maximum variation rate in a domain [4]. Another method [5], based on containing ellipsoids, has been proposed in order to profit from the large amount of data generated in the stochastic search. In all cases, the precise evaluation of sensitivity quantities is intrinsically very time consuming.

In this paper, we present a sensitivity analysis based on a metric directly evaluated from the data provided by MGA, not requiring additional computational effort. This methodology also allows the identification of which solution parameter is more sensitive to deviations.

In the next section, we will present the efficient MGA used; then, we apply the proposed sensitivity methodology, first to minimizing an analytical function (Section III-A), and second to

Digital Object Identifier 10.1109/TMAG.2006.871447

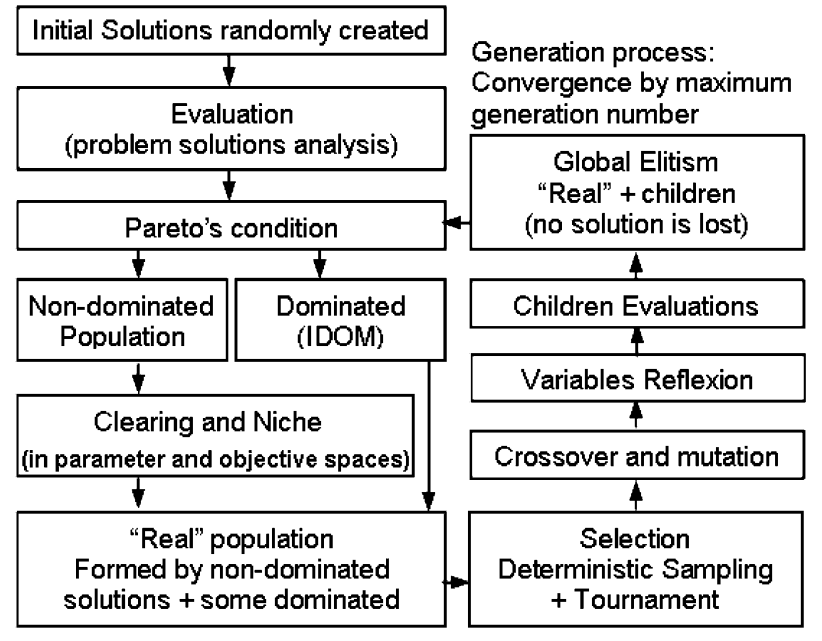

Fig. 1. Block diagram of the Multiobjective Genetic Algorithm.

an electromagnetic problem: the design of a Yagi-Uda Antenna (Section III-B).

\section{Multiobjective Genetic Algorithm}

Founded on the concepts of natural selection and genetics, the MGA optimizer is becoming a well-known stochastic optimization tool in the electromagnetic community [1]-[3]. Fig. 1 shows the procedure used in this work, which benefits from the main improvements published in literature.

Basically, the algorithm starts with a set of solutions randomly created. These solutions are evaluated and the nondominance condition is checked: given a set of $n$ objective functions $f_{i}$ to be minimized $(<)$, one should find the set $P$ of $x *$ solutions defined as

$$
\begin{aligned}
& P=\{x * / \text { it does not exist } x \in S \text { such that: } \\
& \text { 1) there exists } i \text { such that } f_{i}(x)<f_{i}(x *) \text { and } \\
& \text { 2) } \left.\forall i \neq j f_{j}(x) \leq f_{j}(x *)\right\}
\end{aligned}
$$


where $S$ is the feasible search space. The set $P$ contains the efficient solutions $(x *)$ of the problem and it is called the Paretooptimal set.

The result of the Pareto's check (1) is two groups of solutions: one formed by efficient solutions, called the nondominated population (NDOM); and another by nonefficient solutions, called the dominated population (DOM). An index (IDOM) indicating how many times each solution is dominated by others is created. It is important to execute the selection process only with solutions around the Pareto set, which permits a faster convergence. After the Pareto's check, it is time to apply a Clearing technique, the purpose of which is to obtain a sparse and regularly distributed Pareto-front. If similarities among individuals are detected (in parameters or/and objectives spaces [6]), one or some of them are punished. The penalty consists in moving the penalized individual to DOM (by changing IDOM from 0 to 1 ).

We work with a fixed number of individuals (nbind) in the crossover and mutation actions. This fixed minimal size group is called here the real population (REAL), which is recreated at each generation. REAL is composed by all solutions of NDOM (after Clearing); plus $1 / 4 * n$ bind solutions of DOM to maintain some diversity (chosen among those having the smallest IDOM indexes). If the number of REAL's individuals is still less than nbind, we complete with individuals of DOM (choosing other ones with the smallest indexes once again). The opposite will be handled in the selection process.

The number of selected individuals picked up from REAL is always nbind. The selection is done by a mixed method, including Deterministic Sampling and Tournament methods. The parents are in part selected by Deterministic Sampling (based on the population fitness average, which gives emphasis to the Pareto center area) and the remaining by Tournament (based on each objective individually $n$ objectives times, which gives emphasis to the Pareto-edges areas). This approach makes easier the attainment of a well-established Pareto set. After selection, we apply the crossover and mutation operators for real coding schemes [7]. Generation after generation, these operators create new individuals (children) from existing ones (parents), engendering a good "search space" for exploration. Notice that children do not automatically obey all the constraints (e.g., fixed maximal dimensions). In this case, classical techniques are applied: for instance, values above a given limit are reduced using a simple "adjustment by saturation" [1].

The new individuals (children) are evaluated and directly inserted in REAL, from which all solutions are submitted to a nondominance condition evaluation (1). This results in a modified NDOM, whose size fluctuates (increases and decreases), while the DOM can only grow. Similar to a mono-objective GA, new good solutions can appear at any step in the MGA methodology. However, these new individuals can be lost or destroyed at the course of the evolutionary process. The concept of Global Elitism [7] guarantees that efficient solutions will be kept. In the multiobjective algorithm proposed here, the global elitism is implicitly incorporated by the use of all NDOM to compound REAL. Finally, the evolutionary process is restarted with the new DOM and NDOM.

To reduce the computational cost in the evaluation of IDOM, the size of the DOM population is controlled [to keep this

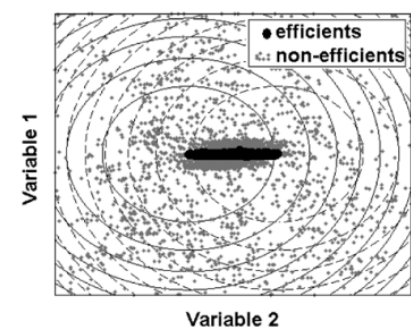

(a)

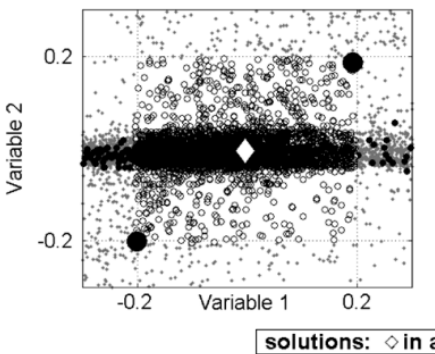

(b)

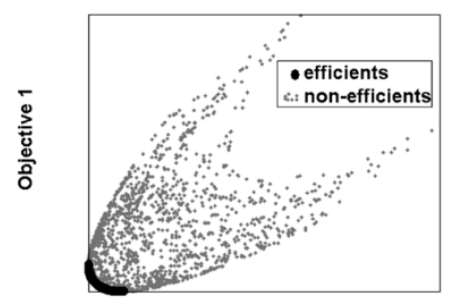

Objective 2

(a')

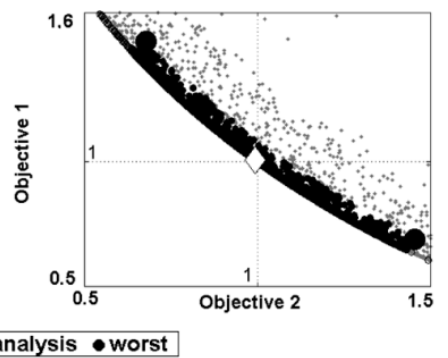

(b’)
Fig. 2. Two parabolas-analytical function test. (a) Parameters space. (a') Pareto-front-Fitness space. (b) Parameters space zoom. (b') Fitness space zoom.

maximum size, some dominated solutions - with a bad IDOM index-are moved to a separate database (DOMold)].

The end of the evolutionary process can be done by some criterion (e.g., maximum number of generations [1]).

\section{MGA With Sensitivity ANALYSis}

The main purpose of the MGA is to search for the Paretofront among all the data generated by the underlying genetic algorithm. If the algorithm does not converge prematurely, the feasible space will be properly represented [6]. As shown in Fig. 2(a), samples are particularly dense around Pareto's region, so that it is reasonable to use this data to implement the sensitivity analysis.

In the first step, we search for the maximum variation in the value of each objective function related to given deviations from the optimal parameter values $\left(V^{*}\right)$. These deviations are specified by the designer to face up to real-world possible perturbations. Afterward, some samples of the Pareto-set are selected to perform the sensitivity analysis. These samples can be some solutions belonging to the Pareto-front used to study the behavior of different regions, or special solutions chosen by some designer preference.

The proposed metric is evaluated using only the already acquired samples inside a perturbation domain around $V^{*}$.

\section{A. Analytical Problem}

Fig. 2 shows the data generated by MGA in the simultaneous minimization of two quadratic objective functions $\left(f_{1}\right.$ and $\left.f_{2}\right)$ with two design variables $\left(x_{1}\right.$ and $\left.x_{2}\right)$

$$
f_{1}=\left(x_{1}-1\right)^{2}+x_{2}^{2} \quad \text { and } \quad f_{2}=\left(x_{1}+1\right)^{2}+x_{2}^{2} .
$$

The optimal region is a line connecting the parabolas' vertexes. 
In this analytical example, we choose the Pareto sample $V^{*}=$ $[-0.003387,-0.004788]$, which is the closest solution to fitness $[1,1]$ found by MGA. We defined the perturbation domain $D^{*}$ as a deviation of $[-0.2,0.2]^{2}$ around $V^{*}$, and then took the sample with the worst fitness inside it. In this example, the worst cases for both objective functions are in the domain corners. The designer considers the solution stable if this worst fitness is acceptable.

To compare how stable is a Pareto sample in relation to others, it is essential to associate the fitness values with the distance in the parameter space. We define the behavior associated with an optimal point $V^{*}$ for each objective function $f_{k}(\cdot)$ inside the perturbation domain $D^{*}$ as

$$
B_{k}\left(V^{*}\right)=\max _{V_{i}, V_{j} \in D^{*}, i \neq j} \frac{\left|f_{k}\left(V_{i}\right)-f_{k}\left(V_{j}\right)\right|}{\left\|V_{i}-V_{j}\right\|}
$$

where $V_{i}$ and $V_{j}$ are samples inside $D^{*}$. Note that $D^{*}$ can be any domain containing $V^{*}$. The evaluation of the behavior is straightforward, since we use only points already sampled. Furthermore, it is a value asymptotically close to the Lipschitz constant as the number of samples grows to infinity, over $D^{*}$. This means that it is an approximation to the maximum mean rate between two points inside $D^{*}$. The greater $B_{k}$ is, the more unstable the function $f_{k}$ at $V^{*}$ will be.

Since distance and objective function variation are positive values in evaluating $B_{k}$ (3), we may normalize them according to the optimal solution in analysis $V^{*}$-normalize each parameter by its respective $V^{*}$ component absolute value, and function variation by $\left|f_{k}\left(V^{*}\right)\right|$-in order to connect directly sensitivity to significant digits. Furthermore, if the variables are too different in range or meaning, the $B_{k}$ may erroneously point out sensitive variables due to the distance in variables space. To overcome this problem, we may normalize each variable by its variability, i.e., the reachable tolerance in variable implementation (e.g., construction precision or variation caused by unavoidable environment conditions). The greater the variability (uncertainty) is, the more sensitive the variable will be.

\section{B. Yagi-Uda Antenna Design}

As a real application for the sensitivity analysis described previously, we designed a five-element (reflector, driver, and three directors) Yagi-Uda antenna. The problem is simple to facilitate comprehension.

The design variables for the implemented MGA are the lengths $l$ and the distances $d$ between the elements. Table I shows the limits of each variable, which defines the optimization feasible search space. At a fixed operation frequency, the design objectives are the maximization of directivity $D$ and maximization of front-to-back ratio (FBR), with the input impedance Zin into the $[45,55] \Omega$ interval as a constraint specification.

The induced emf method [8] is applied to analyze the Yagi-Uda antenna. This method provides a closed-form solution and considers all elements as half-wavelength thin dipoles, so that a sinusoidal current distribution becomes a good approximation. This is fast and reasonably precise if the elements' lengths are close to half-wavelength and the elements' radii
TABLE I

OPTIMIZATION SEARCH SPACE

\begin{tabular}{cccccccc} 
& Limits & Reflector & Feeder & Director 1 & Director 2 & Director 3 \\
\hline \multirow{2}{*}{$l(\lambda)$} & $\min$ & 0.45 & 0.40 & 0.30 & 0.30 & 0.30 \\
\cline { 2 - 8 } & $\max$ & 0.60 & 0.55 & 0.50 & 0.50 & 0.50 \\
\hline \multirow{2}{*}{$d(\lambda)$} & $\min$ & & 0.1 & 0.1 & 0.1 & 0.1 \\
\cline { 2 - 7 } & & 0.25 & 0.5 & 0.5 & 0.5 \\
\hline
\end{tabular}

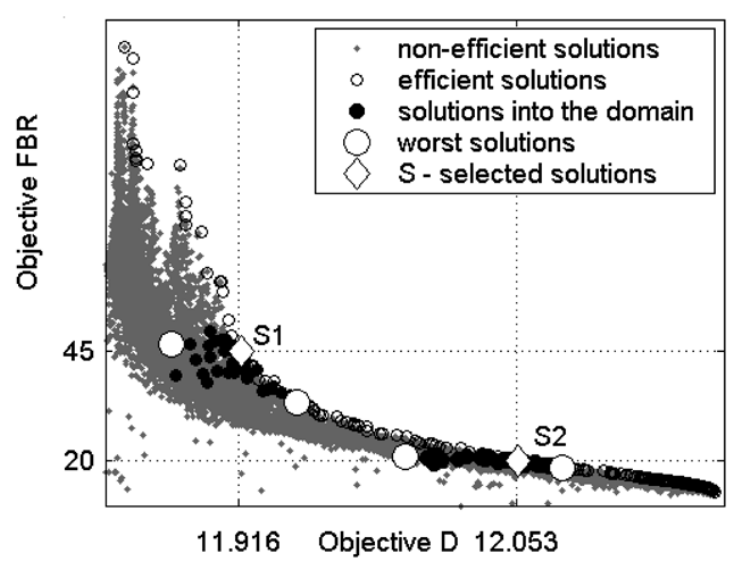

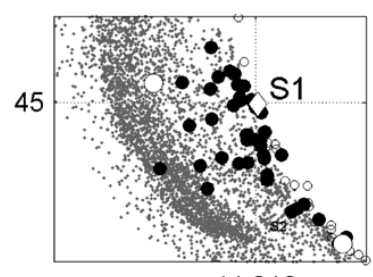

11.916

(a)

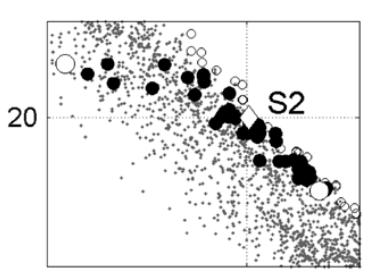

12.053

(b)
Fig. 3. Two parabolas - analytical function test. (a) S1 domain zoom. (b) S2 domain zoom.

are much smaller than both the wavelength $\lambda$ and the distance between elements.

The Pareto-front samples (in gray) and the other ones (in black) are shown in Fig. 3. As an example, we have chosen two solutions (S1 and S2) to perform the sensitivity analysis. The perturbation domain is defined by a $\pm 0.0025 \lambda$ deviation on all design variables.

The samples' studies are presented in Tables II and III. We have solution $\mathrm{S} 1$ with a relatively stable $D$ and an unstable FBR, and $\mathrm{S} 2$, with $D$ and FBR equally stable. Note that in this specific case, the worst objective function value corresponds to the more unstable solution.

The behavior function (3) is upper bounded by the Lipschitz constant (whose numerical evaluation is given in Table III), which would be, in theory, the $B_{k}$ value if we had infinite samples in the domain [4]. Despite some discrepancies, the values provided by the MGA are meaningful as a sensitivity measure.

To point out which solution parameter is more relevant to maintain good device performance, it is enough to perform the behavior analysis on each variable separately. In this case, to create the domain $D^{*}$, we cause a large perturbation on the parameter in analysis and small deviations on the other ones. 
TABLE II

SENSITIVITY ANALYSIS-OPTIMAL PARAMETERS

\begin{tabular}{cccccccc}
\hline & Parameter & Reflector & Feeder & Director 1 & Director 2 & Director 3 \\
\hline S1 & $l(\lambda)$ & 0.48132 & 0.59724 & 0.43109 & 0.42024 & 0.42337 \\
\cline { 2 - 7 } & $d(\lambda)$ & 0.24293 & 0.28425 & 0.33277 & 0.32856 \\
\hline S2 & $l(\lambda)$ & 0.48123 & 0.60380 & 0.42992 & 0.41973 & 0.42333 \\
\cline { 2 - 7 } & $d(\lambda)$ & 0.25216 & 0.29835 & 0.34257 & 0.33225 \\
\hline
\end{tabular}

TABLE III

SENSITIVITY ANALYSIS-OBJECTIVE BEHAVIOR

\begin{tabular}{cccccc}
\cline { 4 - 6 } & \multirow{2}{*}{ Objective } & \multirow{2}{*}{ Solution } & \multirow{2}{*}{ Worst } & \multicolumn{2}{c}{$B_{k}$} \\
\cline { 5 - 6 } & & & MGA & Lipschitz \\
\hline $\mathrm{S}$ & $D(\mathrm{dBi})$ & 11.92 & 11.88 & 44 & 79.5 \\
\cline { 3 - 6 } 1 & $F B R(\mathrm{~dB})$ & 44.90 & 33.40 & 158925 & $23.5 \times 10^{6}$ \\
\hline $\mathrm{S}$ & $D(\mathrm{dBi})$ & 12.05 & 11.99 & 73 & 74.8 \\
\cline { 3 - 6 } 2 & $F B R(\mathrm{~dB})$ & 19.99 & 18.46 & 1485 & 9650 \\
\hline
\end{tabular}

TABLE IV

SENSITIVITY ANALYSIS-S2 PARAMETERS BEHAVIOR

\begin{tabular}{|c|c|c|c|c|c|c|}
\hline$B(\mathrm{l}$ & & Reflector & Feeder & Director 1 & Director 2 & Director 3 \\
\hline \multirow{2}{*}{$D$} & $l$ & 81 & 77 & 73 & 73 & 73 \\
\hline & $d$ & 97 & & 170 & 73 & 12 \\
\hline \multirow{2}{*}{$F B R$} & $l$ & 1544 & 1499 & 1485 & 1485 & 1485 \\
\hline & $d$ & 1519 & & 2640 & 485 & 740 \\
\hline
\end{tabular}

Keeping S2 as an example (with $\pm 0.005 \lambda$ deviation for each variable in analysis and $\pm 0.0025 \lambda$ for the others), the worst situation occurs for the distance between the feeder and the first director parameter (170-2640), followed by the distance between the second and the last director one (112-1740). In other words, these two are the variables that degrade the antenna performance most. The other parameters have almost the same importance concerning the solution stability. Table IV displays the values.

The proposed methodology gives us a satisfactory notion of sensitivity; without any additional computational effort (new evaluations of the problem), it is possible to determine which solution maintains its characteristics in spite of real-world perturbations.

Furthermore, like in all stochastic optimization procedures [1], it is often required to repeat the optimization process to guarantee the correct result (here, the accuracy of the Pareto-front). These repetitions only improve the sensitivity study proposed here; we can use for it all the samples computed during the successive runs.
The analysis method described here uses direct variables. In the case of indirect optimization parameters, such as series coefficients, the proposed analysis could not have real meaning. In that case, it may be necessary to create a parameterization for the perturbation, related to that used in the optimization process, in order to continue using the samples. It would also be possible to work in a reverse approach (without using such parameterization), accepting a percentage deviation for each objective as tolerable; a finite domain of solutions would be defined around the selected ones. We search into this domain for the solution that has the maximal distance to the set extremes; this is the most stable solution.

\section{CONCLUSION}

This paper presents an easy way to perform the multiobjective optimization with sensitivity analysis. This is important in order to consider the real-world design problems that cannot be well controlled by the designer. The procedure is not exact, but it gives us a satisfactory notion of sensitivity. Moreover, no additional computation effort is necessary if the optimization stochastic method works well (allowing a good exploration of feasible search space).

\section{ACKNOWLEDGMENT}

This work was supported in part by the CAPES, CNPq, and CNRS Foundations.

\section{REFERENCES}

[1] C. A. Coello Coello, D. A. Van Veldhuizen, and G. B. Lamont, Evolutionary Algorithms for Solving Multi-Objective Problems (Genetic Algorithms and Evoluationary Computation). Norwell, MA: Kluwer, May 2002.

[2] J. L. Coulomb and L. Lebensztajn, "TEAm workshop problem 25: A multiobjective analysis," IEEE Trans. Magn., vol. 40, no. 2, pp. 1402-1405, Mar. 2004.

[3] P. Di Barba and M. E. Mognaschi, "Recent experiences of multi-objective optimization in electromagnetics: A comparison of methods," presented at the 8th Int. Workshop on Optimization and Inverse Problems in Electromagnetism, Grenoble, France, Sep. 2004.

[4] D. A. G. Vieira et al., "Multi-objective sensitivity analysis in finite domains of a Yagi-Uda antenna optimal design," presented at the 11th IEEE Conf. Electromagnetic Field Computation, Seoul, Korea, Jun. 2004.

[5] R. H. C. Takahashi et al., "Sensitivity analysis for optimization problems solved by stochastic methods," IEEE Trans. Magn., vol. 37, no. 4, pp. 3566-3569, Sep. 2001.

[6] S. L. Avila et al., "The niche technique in parameters and fitness space for multi-objective genetic algorithm optimization," presented at the 11th Int. IGTE Symp. Numerical Field Calculation in Electrical Engineering, Graz, Austria, Sep. 2004

[7] S. L. Avila et al., "Modified genetic operators for multi-objective optimization problems," presented at the 11th IEEE Conf. Electromagnetic Field Computation, Seoul, Korea, Jun. 2004.

[8] C. A. Balanis, Antenna Theory: Analysis and Design. New York: Wiley, 1996.

Manuscript received June 20, 2005 (e-mail: avila@grucad.ufsc.br). 